\title{
Very High Radiation Detector for the LHC BLM System Based on Secondary Electron Emission
}

\author{
B. Dehning; G. Ferioli; E.B. Holzer, D. Kramer. \\ CERN - Geneva/CH
}

\begin{abstract}
Beam Loss Monitoring (BLM) system plays a vital role in the active protection of the LHC accelerators elements. It should provide the number of particles lost from the primary hadron beam by measuring the radiation field induced by their interaction with matter surrounding the beam pipe. The LHC BLM system will use ionization chambers as standard detectors but in the areas where very high dose rates are expected, the Secondary Emission Monitor (SEM) chambers will be employed because of their high linearity, low sensitivity and fast response.

The SEM needs a high vacuum for proper operation and has to be functional for up to 20 years, therefore all the components were designed according to the UHV requirements and a getter pump was included. The SEM electrodes are made of Ti because of its Secondary Emission Yield (SEY) stability. The sensitivity of the SEM was modelled in Geant4 via the Photo-Absorption Ionization module together with custom parameterization of the very low energy secondary electron production. The prototypes were calibrated by proton beams in CERN PS Booster dump line, SPS transfer line and in PSI Optis line. The results were compared to the simulations.
\end{abstract}

Paper presented at the IEEE 2007 Conference - Honolulu/USA $-27^{\text {th }}$ October- $3^{\text {rd }}$ November 2007 


\title{
Very High Radiation Detector for the LHC BLM System Based on Secondary Electron Emission
}

\author{
Daniel Kramer, Bernd Dehning, Eva Barbara Holzer, Gianfranco Ferioli
}

\begin{abstract}
Beam Loss Monitoring (BLM) system plays a vital role in the active protection of the LHC accelerators elements. It should provide the number of particles lost from the primary hadron beam by measuring the radiation field induced by their interaction with matter surrounding the beam pipe. The LHC BLM system will use ionization chambers as standard detectors but in the areas where very high dose rates are expected, the Secondary Emission Monitor (SEM) chambers will be employed because of their high linearity, low sensitivity and fast response. The SEM needs a high vacuum for proper operation and has to be functional for up to 20 years, therefore all the components were designed according to the UHV requirements and a getter pump was included. The SEM electrodes are made of Ti because of its Secondary Emission Yield (SEY) stability. The sensitivity of the SEM was modeled in Geant4 via the Photo-Absorption Ionization module together with custom parameterization of the very low energy secondary electron production. The prototypes were calibrated by proton beams in CERN PS Booster dump line, SPS transfer line and in PSI Optis line. The results were compared to the simulations.
\end{abstract}

Index Terms-beam loss monitor, secondary emission, LHC, beam instrumentation.

\section{BLM SYSTEM}

$\mathbf{T}$ HE Beam Loss Monitoring system [1] is a vital part of the active LHC machine protection. It has to detect dangerous beam losses which could quench the superconductive magnets or even damage components of the accelerator. 3700 ionization chambers (BLMI) will be used in LHC as the main beam loss detectors. (i.e. each quadrupole magnet has 6 monitors installed).

Additional 280 BLMS detectors are needed for increasing the dynamic range of the BLM system in the areas, where very high foreseen or unforeseen losses might occur. This concerns mainly the collimation zones, injection points, interaction points, beam dump and at other critical aperture limits (i.e. every collimator has one BLMS and one BLMI installed on its support).

\section{BLMS DETECTOR}

The detector has to keep a linear response for very high particle fluxes, so it has to have a high saturation limit. High stability of the response and radiation tolerance is needed, because large fluencies up to $70 \mathrm{MGy} /$ year might be integrated during the nominal LHC operation. It will be nearly impossible

D. Kramer is the corresponding author (e-mail: daniel.kramer@cern.ch). All authors are with CERN, CH-1211 Geneva 23, Switzerland (e-mails: firstname.lastname@cern.ch).

D.Kramer is also with Technical university of Liberec, Hálkova 6, 46117 Liberec 1, Czech Republic (e-mail: daniel.kramer@tul.cz)

Manuscript received November 16, 2007. to exchange the BLMS in some locations (like under the core of the beam dumps), therefore the lifetime should be 20 years as predicted for the LHC.

The detector will be connected to the same measurement system [11] as the BLMI but should extend the dynamic range of the setup from $10^{8}$ to $10^{13}$. Consequently, the BLMS must have a lower response than the ionization chambers. The absolute sensitivity should be such that several hundreds of $\mathrm{nA}$ would correspond to the dose rate of $22 \mathrm{mGy} / \mathrm{s}$, which is the saturation limit of the electronics with the BLMI (i.e. $1 \mathrm{~mA})$.

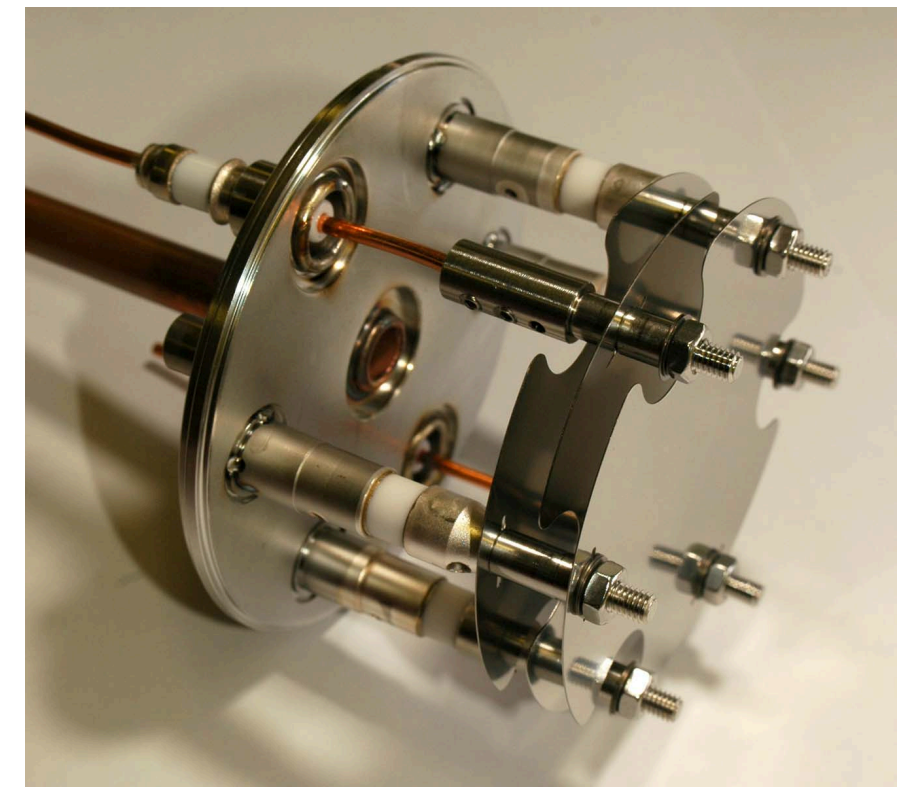

Fig. 1. SEM detector assembly before welding of the vacuum vessel containing a NEG pump.

\section{A. SEM working principle}

The BLMS detector is based on the Secondary Electron (SE) emission from metallic surfaces and so it is called the SEM (Secondary Emission Monitor). When a charged particle passes through the signal electrode, it excites conduction band and inner shell electrons. These electrons can diffuse only several $\mathrm{nm}$ as they usually have energies lower than $50 \mathrm{eV}$ independent of the primary particle's energy and type [2] in contrary to the "knock-on" $\delta$ electrons. The material escaping SE come only from a thin surface layer of the traversed material and are subsequently drifted away by a bias electric field. The Secondary electron Emission Yield (SEY) 
is proportional to the electronic energy loss of the particle in the surface layer of the signal electrode. The current created by the drifting electrons is measured between the signal and the bias electrodes.

The "high" energy $\delta$ electrons are produced mostly in the forward direction (same as the primary particle). If they are emitted from the signal electrode, their contribution is in average canceled by the $\delta$ electrons arriving from the bias electrode. Nevertheless when the $\delta$ electrons have enough energy to penetrate the electrodes, they will create current only if were emitted from the signal electrode.

The SEM can detect neutral particles only indirectly. The neutral particles have to interact with any part of the detector and create charged secondaries, which can be detected. Generally, the charged particles can also produce a signal if their path lengths in the two bias field gaps are not equal.

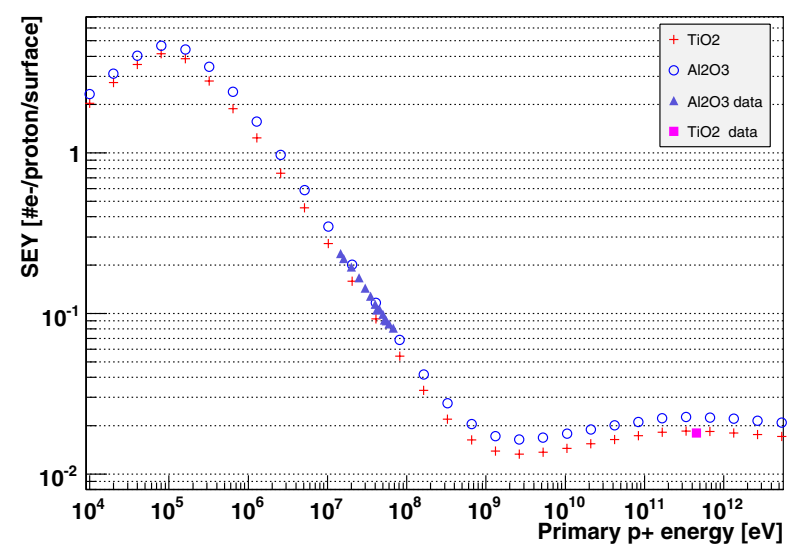

Fig. 2. Modified Sternglass formula for true SEY of primary protons for different materials scaled by factor 0.8 to fit reference data[6], [7].

\section{B. Detector design}

The detector was designed with respect to the UHV requirements and materials with low radiation induced outgassing were chosen (i.e. Ti instead of $\mathrm{Al}$ ). The electrodes are made of $0.25 \mathrm{~mm}$ thick $\mathrm{Ti}$, because its SEY was found to be stable up to $10^{20} p^{+} / \mathrm{cm}^{2}$ integrated dose [5]. The detector has to operate in high vacuum of at least $10^{-4} \mathrm{mbar}$, because the contribution of the gas ionization to the signal has to be kept below $1 \%$ of the secondary emission to prevent a nonlinear response. All the steel components undergo the standard CERN UHV cleaning procedure and are vacuum fired at $950^{\circ} \mathrm{C}$ for several hours. This procedure eliminates the hydrogen dissolved inside the steel. The Ti parts are fired only at $750^{\circ} \mathrm{C}$ to prevent the pollution from the oven and phase change of the material. A careful insulation of the signal path outside of the detector was found to be very important to prevent a signal contribution from the ionization in air. The response is otherwise nonlinear and strongly depends on the applied bias voltage.

Due to the radiation induced and thermal outgassing, the pressure inside the detector could not be maintained without active pumping. The detector contains a Non Evaporable
Getter (NEG) St707 [10] foil of $170 \mathrm{~cm}^{2}$ active area inside the steel vessel. Its total absorption capacity of $\mathrm{H}_{2}$ and $\mathrm{CO}$ is higher than the equivalent of one monolayer covering the inner surfaces. The NEG is activated during a $350^{\circ} \mathrm{C}$ part of the bakeout cycle.

The outgassing of the detector without the NEG was tested and a slow pumping of the Ti was observed. The pressure inside the detector after the NEG activation and before the pinch-off is below $10^{-10}$ mbar.

\section{MODELING OF BLMS RESPONSE}

\section{A. SEY estimation}

There is no Secondary Electron Emission process in Geant4 [3] up to now, so a custom generator was created. A modified semi empirical formula of Sternglass [4] (the contribution of $\delta$ electrons to the true SEY was not included) was used to calculate the SEY for the $\mathrm{TiO}_{2}$ surface.

$$
S E Y=\left.0.01 C_{F} L_{S} \frac{d E}{d x}\right|_{e l} \quad L_{S}=\left(0.23 N \sigma_{g}\right)^{-1}
$$

Where $d E /\left.d x\right|_{e l}$ stands for electronic energy loss, $L_{S}$ for effective penetration distance of SE, $\mathrm{N}$ for number of atoms per unit volume and $\sigma_{g}=1.6 Z^{1 / 3} 10^{-16} \mathrm{~cm}^{2}$. The calibration factor $C_{F}=0.8$ was used in order to match the experimental data for $\mathrm{Al}_{2} \mathrm{O}_{3}$ [6] and $\mathrm{TiO}_{2}$ [7]. The maximum measured SEY for the very low energy (i.e. $100 \mathrm{keV}$ ) protons hitting the $\mathrm{Al}$ target is 1.3 [8] (not plotted) compared to 2 from the parametrization, but particles with such energies have a negligible contribution to the signal as they don't penetrate the chamber walls or lie below the $e^{-}$production cut of the simulation. The resulting functional dependence for different materials can be found in Figure 2.

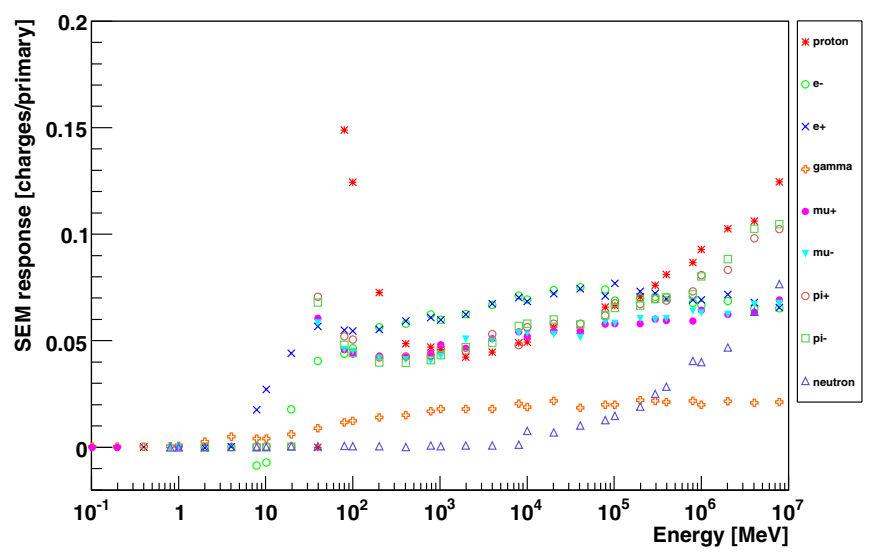

Fig. 3. Simulated response curves for different particle types and wide energy range.

\section{B. Geant4 simulations}

A detailed geometry of the SEM prototype was implemented in Geant4 including a thin layer of $\mathrm{TiO}_{2}$ on the signal electrode. The signal is generated in two ways in 
the G4UserSteppingAction. When a charged particle passes through the $\mathrm{TiO}_{2}$ to vacuum interface, the SEY is calculated using the Eq. III-A and a SE is recorded with the corresponding probability. The SE can be generated also from other the metallic surfaces connected to the signal electrode. The $d E /\left.d x\right|_{e l}$ is calculated by the G4EmCalculator but in case of primary $e^{-}$or $e^{+}$, the $\mathrm{dE} / \mathrm{dx}$ from Bremsstrahlung must be subtracted and for $m u^{-}$or $m u^{+}$also the $e^{-} / e^{+}$ pair production, as these processes don't contribute directly to secondary emission. Nevertheless, their products are treated as other particles.The second part of the signal is obtained simply by calculating the charge balance on the signal electrode.

The $\delta$ electrons are produced by the Photo-Absorption Ionization (PAI) module [12] and are treated as other charged particles. The Geant4 QGSP_BERT_HP was used as the main physics list. The production threshold for electrons was set to $9 \mu \mathrm{m}$. The error bars of the simulations are produced only from the statistical fluctuations.

\section{Response for different particle types}

The detector will be used mainly in the mixed radiation fields of particle showers (i.e. after the collimators) created by the lost primary protons. It is therefore necessary to predict the response for a wide energy and particle type range. These curves will be used for generating the beam abort request thresholds. The Figure 3 shows the result of simulations using Geant4.8.1.p01 and a round gaussian beam impacting through the center of the bottom plate. When a primary particle has just the right energy to get fully absorbed inside the signal electrode, the output is dominated by the charge balance and can be negative as seen for the electrons of approx. $8 \mathrm{MeV}$. The charged hadrons show a relatively sharp threshold behavior caused by the Bragg peak laying inside the steel cover. The particles with slightly more energy arrive to the signal electrode with a low residual energy thus having a high $\mathrm{dE} / \mathrm{dx}$. The signal rise for hadrons at high energies is caused by the hadronic showers initiated in different parts of the detector.

\section{Measurements}

The simulations are validated by measurements with particle beams of known parameters. Different prototypes were placed directly in the extracted primary proton beams in the Paul Scherrer Institute (PSI), in CERN PS Booster dump line and SPS transfer line.

\section{A. Calibration with $63 \mathrm{MeV}$ protons}

The design of the version "C" is very similar to the BLMI, it contains a large ceramic disk holding the supports of the electrodes. The version "F" looks very similar to the final design from Figure 1, only the electrode holders are slightly different and it does not contain any NEG.

These prototypes were tested in the $62.9 \mathrm{MeV}$ proton Optis line in PSI [9]. Protons were entering through the $5 \mathrm{~mm}$ thick steel bottom cover of the detector. The output current was measured by a Keithley electrometer 6517A. The bias high voltage was varied from $2 \mathrm{~V}$ to $1.5 \mathrm{kV}$ and the resulting

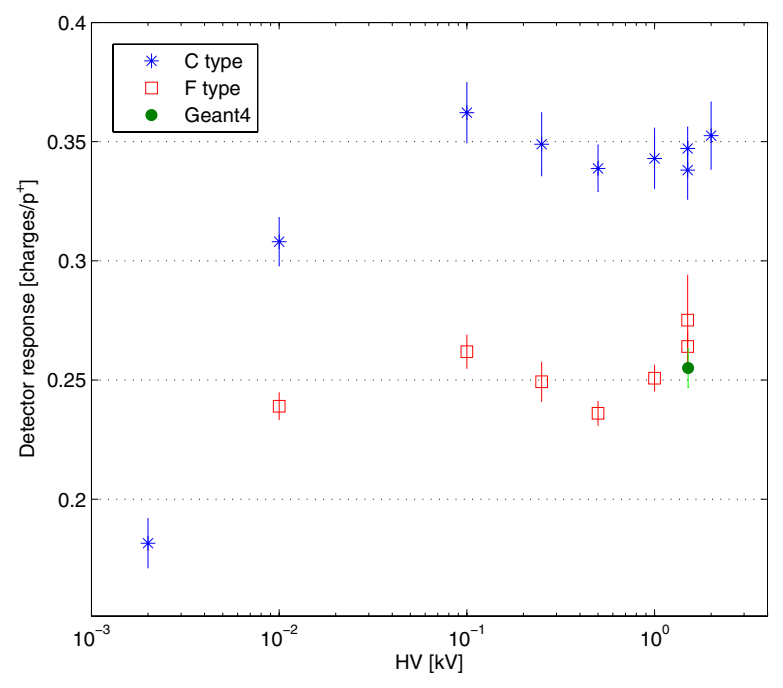

Fig. 4. Variation of BLMS normalized response with bias voltage for two prototype detector versions (63 MeV cyclotron proton beam in PSI).

SEY was calculated by dividing the beam current by the detector output. Figure 4 shows a systematic pattern which seems to be caused by the low energy $\delta$ electrons coming from the HV electrodes. This variation was not reproduced by the simulations, partly due to the higher production threshold. The corresponding simulations were performed with a $1.5 \mathrm{kV}$ electric field and are in a good agreement with measurements for the "F type".

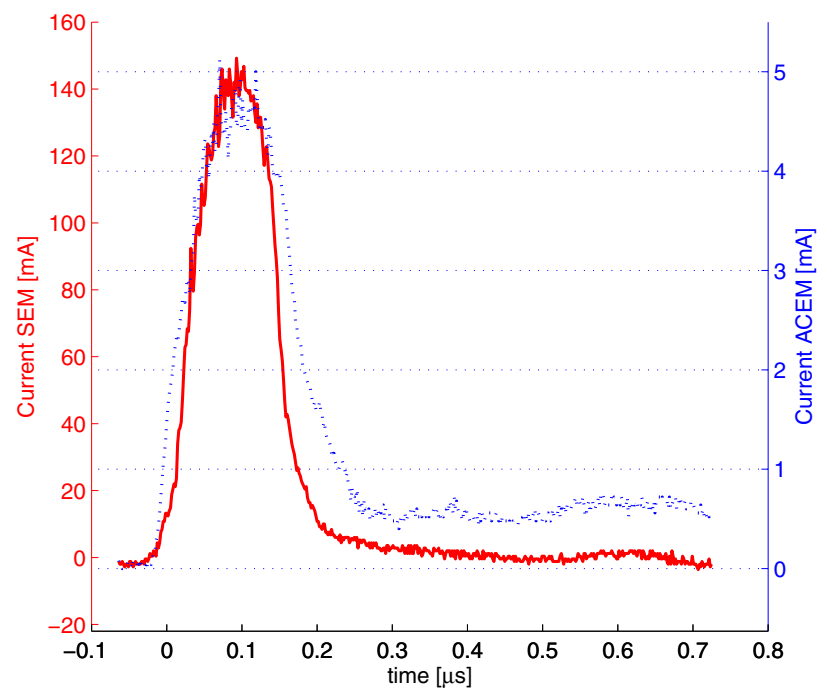

Fig. 5. Response to single bunch passage compared to reference ACEM detector $\left(160 \mathrm{~ns}\right.$ bunch of $1.1 \times 10^{11} p^{+}$at $\left.1.4 \mathrm{GeV}\right)$.

\section{B. Calibration with $1.4 \mathrm{GeV}$ protons}

The older "prototype C" was also installed in the PS Booster dump line and tested with a bunched proton beam. A reference ACEM (Aluminum Cathode Electron Multiplier tube) detector 


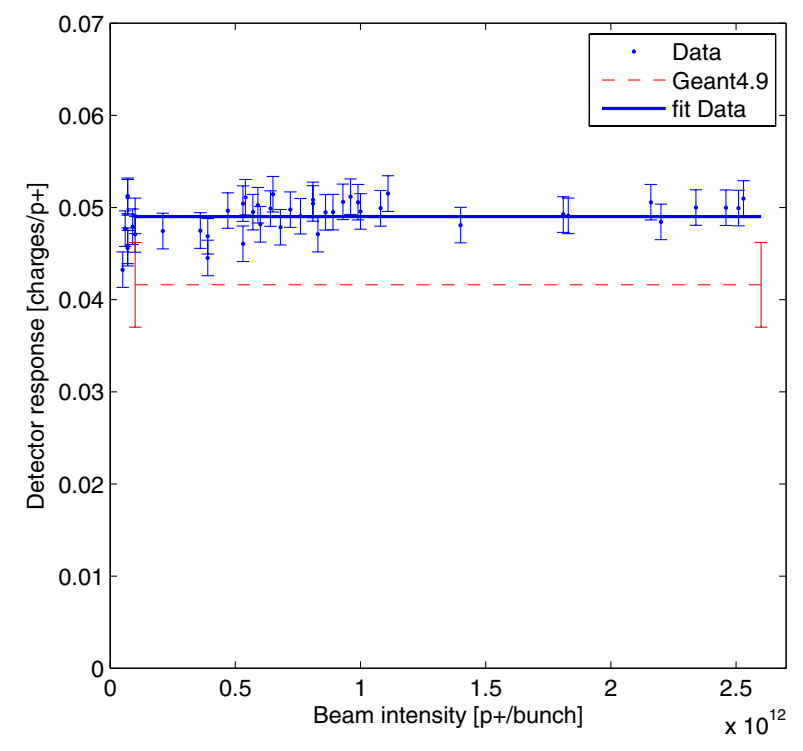

Fig. 6. SEY of BLMS as function of proton beam intensity at $1.4 \mathrm{GeV}$. Simulation error was estimated to $10 \%$.

with a fast response time was installed close to the SEM outside of the beam. On the Figure 5, the SEM measured the same bunch shape as the ACEM while the longitudinal bunch length was about 160ns. The chamber signals were measured with Tektronics oscilloscope and $50 \Omega$ termination. The integration was done offline in a Matlab code.

Figure 6 shows a very good linearity of the SEM and a reasonable agreement with the simulation. The measured prototype was not implemented in Geant4 but the "prototype F" was used instead. From the measured difference in Figure 4, one would expect a $25 \%$ underestimation, which is likely the case.

\section{Calibration with $400 \mathrm{GeV}$ protons}

The "prototype F" was installed in the SPS TT20 transfer line. It was placed on a movable stage between two vacuum sections of the beam pipe. The transverse profile of the proton beam was estimated to about $2 \mathrm{~mm}$ rms and a conservative misalignment of $1^{\circ}$ with respect to the detector axis was assumed. The Figure 7 shows the result of the measurements together with the simulation. Each point represents one slow extraction passing through the bottom of the detector transverse to the surface of the electrodes.

The negative signal peaks are created when the beam passes through (and parallel to) the detector walls and produces a large number of low energy secondaries, which hit the signal electrode only coming from the side.

\section{Conclusions}

The prototypes of the SEM beam loss detector were extensively tested and calibrated in different proton beams. The modeling approach in Geant 4 was developed and validated by correctly reproducing the experiments. The design of the detector was finalized and the vacuum properties were measured. Negative signals were also observed and partially simulated,

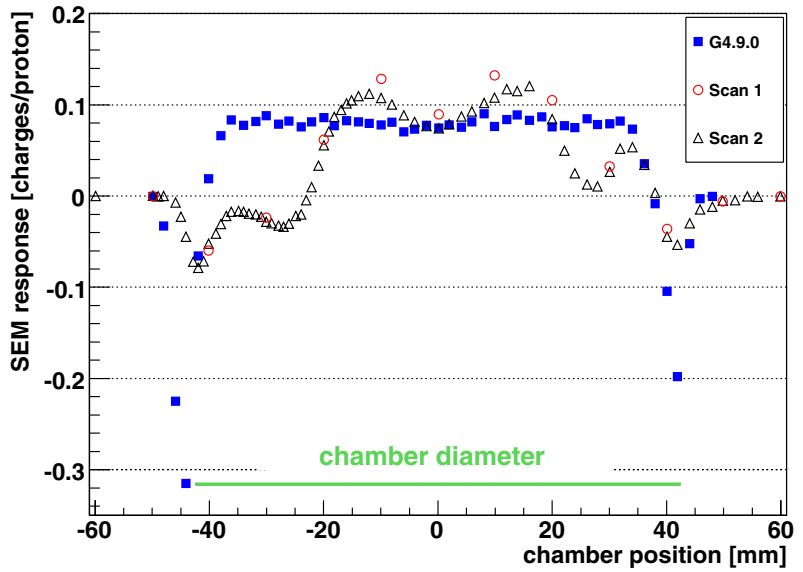

Fig. 7. Simulation and measurement of SEM moved through $400 \mathrm{GeV}$ proton beam.

but can occur only under very special circumstances.

All the chambers were or will be measured in a fixed target test beam at CERN. The LHC BLM system verification tests including the SEMs placed after the LHC prototype collimator in the SPS are ongoing.

\section{ACKNOWLEDGMENT}

The authors would like to thank to Paolo Chiggiatto and Ivo Wevers for their help in designing and testing the vacuum properties of the SEM. The collaboration with IHEP Protvino, where 360 detectors were successfully produced is also acknowledged.

\section{REFERENCES}

[1] E.B. Holzer et al., Beam Loss Monitoring System for the LHC, IEEE NSS '05, Puerto Rico, CERN-AB-2006-009 BI.

[2] D. Hasselkamp et al., Particle Induced Electron Emission II, SpringerVerlag, (1992).

[3] Geant4 - A Simulation Toolkit, S. Agostinelli et al., Nuclear Instruments and Methods A 506 (2003) 250-303.

[4] E.J. Sternglass, Theory of Secondary Electron Emission by High-Speed Ions, Phys. Rev. 108(1957) 1.

[5] G. Ferioli and R. Jung, Evolution of the Secondary Emission Efficiencies of various materials measured in the CERN SPS secondary beam lines, CERN-SL-97-071-BI, (1997).

[6] C.M. Castaneda et al., Secondary electron yields from the bombardment of $\mathrm{Al}_{2} \mathrm{O}_{3}$ by protons, deuterons, alpha particles and positively charged hydrogen molecules at energies in the range of 10 to $80 \mathrm{MeV}$, Nuclear Instr. and Meth. B 129(1997) 199-202.

[7] K. Bernier et al., Calibration of secondary emission monitors of absolute proton beam intensity in the CERN SPS North Area, CERN-97-07, (1997).

[8] B. Svensson and G. Holmén, Electron Emission from aluminum and copper under molecular-hydrogen-ion bombardment, Phys. Rev. B 25(1982) 5.

[9] Paul Scherrer Institute, Villigen Switzerland, http://www.psi.ch.

[10] C. Benvenuti and P. Chiggiato, Pumping characteristics of the St707 non-evaporable getter ( $\mathrm{Zr} 70-\mathrm{V}$ 24.6-Fe $5.4 \mathrm{wt} \%)$, J. Vac. Sci. Technol. A 14 (1996) 3278-3282.

[11] C. Zamantzas et al., The LHC Beam Loss Monitoring System's Data Contribution to other Systems, IEEE NSS '07, Honolulu Hawaii, These proceedings.

[12] V.M. Grichine et al., An implementation of ionisation energy loss in very thin absorbers for the GEANT4 simulation package, Nucl. Instrum. Methods Phys. Res., A 453 (2000) 597-605. 